\section{AUTHOR CONTRIBUTIONS}

$\mathrm{CT}$ designed the research, analyzed the data and wrote the paper; IS supervised the work; PVR, SC, RP and CDB performed experiments; GZ provided AML cell lines, reagents and analyzed the data; SC and DB commented on the paper. SC and RF provided AML samples. AA provided T-ALL samples. CT, PVR, SC assembled the figures.

\section{AUTHOR CONTRIBUTIONS}

$\mathrm{CT}$ designed the research, analyzed the data and wrote the paper; IS supervised the work; PVR, SC, RP and CDB performed experiments; GZ provided AML cell lines, reagents and analyzed the data; SC and DB commented on the paper. SC and RF provided AML samples. AA provided T-ALL samples. CT, PVR, SC assembled the figures.

P Vargas Romero ${ }^{1}$, S Cialfi $^{1}$, R Palermo ${ }^{2}$, C De Blasio ${ }^{1}$, S Checquolo ${ }^{3}$, D Bellavia ${ }^{1}$, S Chiaretti ${ }^{4}$, R Foà ${ }^{4}$, A Amadori ${ }^{5}$, A Gulino ${ }^{1,6,7}$, G Zardo ${ }^{4}$, C Talora ${ }^{1}$ and I Screpanti ${ }^{1}$

${ }^{1}$ Department of Molecular Medicine, Sapienza University of Rome, Rome, Italy;

${ }^{2}$ Center for Life Nano Science@Sapienza, Istituto Italiano di Tecnologia, Rome, Italy;

${ }^{3}$ Department of Biotechnology and Medical-Surgical Sciences, Sapienza University, Latina, Italy; ${ }^{4}$ Department of Cellular Biotechnologies and Hematology, Sapienza University of Rome, Rome, Italy;

${ }^{5}$ Department of Surgery, Oncology and Gastroenterology, University of Padua, Padua, Italy and

${ }^{6}$ Neuromed Institute, Pozzilli, Italy E-mail: claudio.talora@uniroma1.it or isabella.screpanti@uniroma1.it ${ }^{7}$ Dedicated to the cherished memory of Alberto Gulino.

\section{REFERENCES}

1 Bartel DP. MicroRNAs: target recognition and regulatory functions. Cell 2009; 136: 215-233.

2 Giambra V, Jenkins CR, Wang H, Lam SH, Shevchuk OO, Nemirovsky O et al. NOTCH1 promotes $\mathrm{T}$ cell leukemia-initiating activity by RUNX-mediated regulation of PKC-theta and reactive oxygen species. Nat Med 2012; 18: 1693-1698.

3 Bousquet M, Harris MH, Zhou B, Lodish HF. MicroRNA miR-125b causes leukemia. Proc Natl Acad Sci USA 2010; 107: 21558-21563.

4 Shaham L, Binder V, Gefen N, Borkhardt A, Izraeli S. MiR-125 in normal and malignant hematopoiesis. Leukemia 2012; 26: 2011-2018.

5 Sun YM, Lin KY, Chen YQ. Diverse functions of miR-125 family in different cell contexts. J Hematol Oncol 2013; 6: 6.

6 Tili E, Michaille JJ, Croce CM. MicroRNAs play a central role in molecular dysfunctions linking inflammation with cancer. Immunol Rev 2013; 253: 167-184.

7 So AY, Zhao JL, Baltimore D. The Yin and Yang of microRNAs: leukemia and immunity. Immunol Rev 2013; 253: 129-145.

8 Nerlov C. C/EBPalpha mutations in acute myeloid leukaemias. Nat Rev Cancer 2004; 4: 394-400.

9 Ohlsson E, Hasemann MS, Willer A, Lauridsen FK, Rapin N, Jendholm J et al. Initiation of MLL-rearranged AML is dependent on C/EBPalpha. J Exp Med 2014; 211: 5-13.

10 Roe JS, Vakoc CR. C/EBPalpha: critical at the origin of leukemic transformation. J Exp Med 2014; 211: 1-4.

11 Cialfi S, Oliviero C, Ceccarelli S, Marchese C, Barbieri L, Biolcati G et al. Complex multipathways alterations and oxidative stress are associated with Hailey-Hailey disease. Br J Dermatol 2010; 162: 518-526.

12 Manca S, Magrelli A, Cialfi S, Lefort K, Ambra R, Alimandi M et al. Oxidative stress activation of miR-125b is part of the molecular switch for Hailey-Hailey disease manifestation. Exp Dermatol 2011; 20: 932-937.

13 Lobry C, Oh P, Mansour MR, Look AT, Aifantis I. Notch signaling: switching an oncogene to a tumor suppressor. Blood 2014; 123: 2451-2459.

14 Katzerke C, Madan V, Gerloff D, Brauer-Hartmann D, Hartmann JU, Wurm AA et al. Transcription factor C/EBPalpha-induced microRNA-30c inactivates Notch1 during granulopoiesis and is downregulated in acute myeloid leukemia. Blood 2013; 122: 2433-2442.

15 Benelli D, Cialfi S, Pinzaglia M, Talora C, Londei P. The translation factor elF6 is a Notch-dependent regulator of cell migration and invasion. PLoS One 2012; 7: e32047.

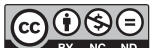

This work is licensed under a Creative Commons AttributionNonCommercial-NoDerivs 4.0 International License. The images or other third party material in this article are included in the article's Creative Commons license, unless indicated otherwise in the credit line; if the material is not included under the Creative Commons license, users will need to obtain permission from the license holder to reproduce the material. To view a copy of this license, visit http:// creativecommons.org/licenses/by-nc-nd/4.0/

\title{
A novel recurrent EP300-ZNF384 gene fusion in B-cell precursor acute lymphoblastic leukemia
}

\section{Leukemia (2015) 29, 2445-2448; doi:10.1038/leu.2015.111}

In pediatric patients with B-cell precursor acute lymphoblastic leukemia (BCP-ALL), approximately three-quarters harbor wellcharacterized, clinically relevant chromosomal alterations, including hyperdiploidy, hypodiploidy, $\mathrm{t}(12 ; 21)$ ETV6/RUNX1, $\mathrm{t}(1 ; 19) E 2 A /$ $P B X 1, \mathrm{t}(9 ; 22) B C R / A B L 1$ and the rearrangement of $M L L$ at $11 \mathrm{q} 23$, and they can facilitate diagnosis, risk stratification and targeted therapy. ${ }^{1,2}$ In the remaining patients, however, major pathogenic or driver gene abnormalities and their association with the clinical outcome have yet to be fully clarified. As recent advanced genomic studies using next-generation sequencing have identified a number of novel fusion genes and stratified a high-risk subtype in BCP-ALL, ${ }^{3-5}$ unknown genetic alterations that constitute characteristic subgroups may still exist in the remaining patients. We therefore intended to investigate unknown fusion genes in BCP-ALL by using next-generation sequencing.
As a consequence of whole transcriptome sequencing performed on complementary DNA from 55 selected samples of pediatric BCP-ALL patients without conventional genetic abnormalities (Supplementary Information), an EP300-ZNF384 fusion gene was identified in two patients (Cases 1 and 2) as a repeatable and plausible candidate fusion gene (Figure 1a). The 372-bp fragment of the EP300-ZNF384 fusion CDNA was amplified by RT-PCR using a pair of specific primers, and Sanger sequencing of the PCR products revealed a sequence of the products identical to that obtained by whole transcriptome sequencing (Supplementary Figure 1). The presence of EP300-ZNF384 fusion in Case 1 was further confirmed by FISH using a combination of appropriate probes for EP300 and ZNF384, respectively (Figure 1b). We screened a further 346 of pediatric ALL cases by RT-PCR, and identified 4 additional patients with EP300-ZNF384 fusion (Cases 3-6, Supplementary Figure 1). All six patients were BCP-ALL without conventional cytogenetic abnormalities. Our RNA samples 

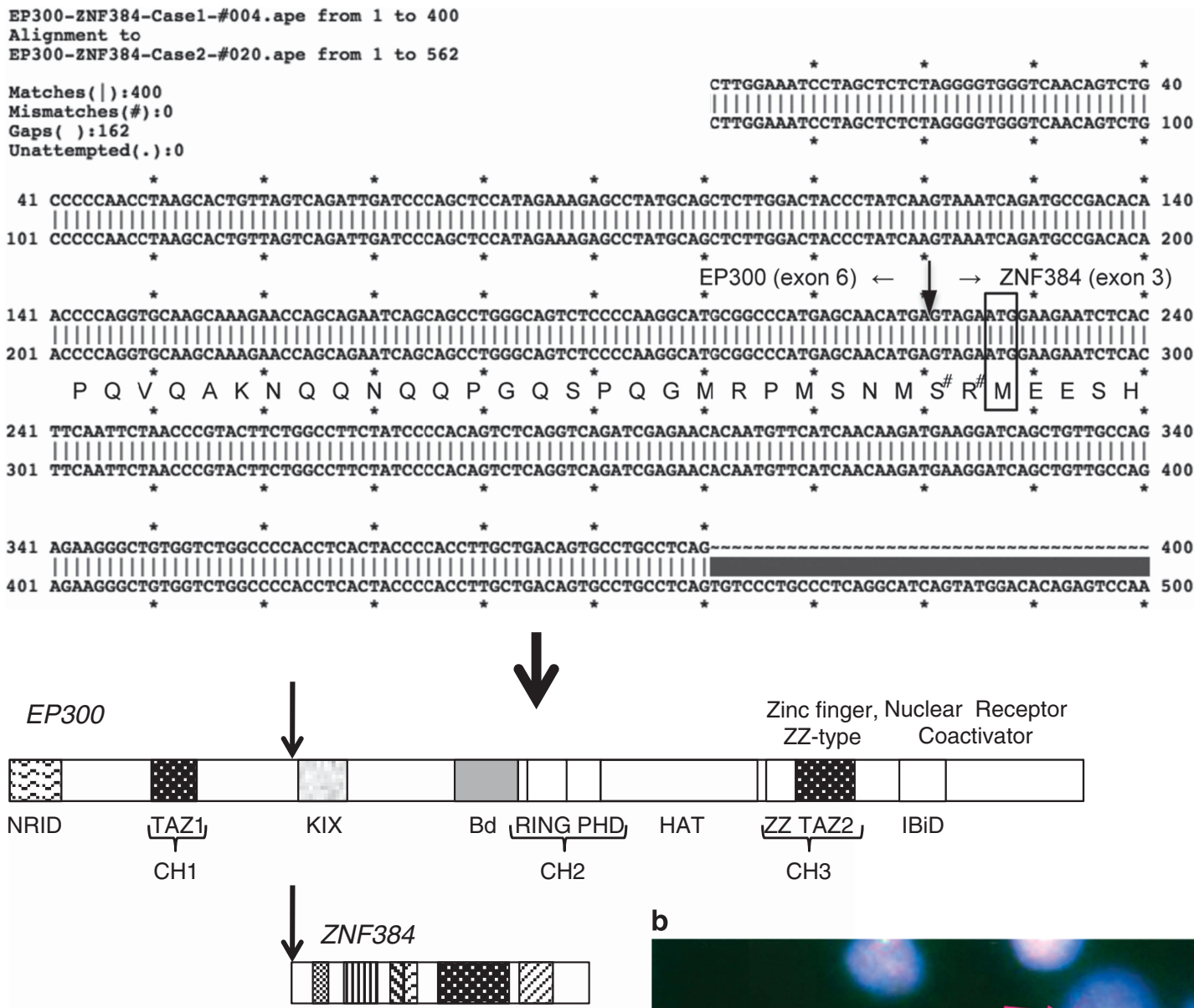

LZ SRRRNLS ZFS QA

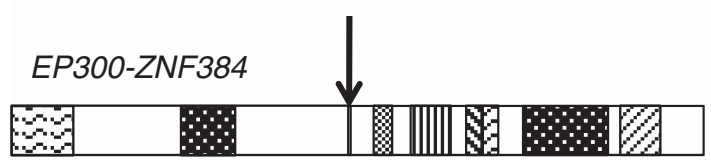

b

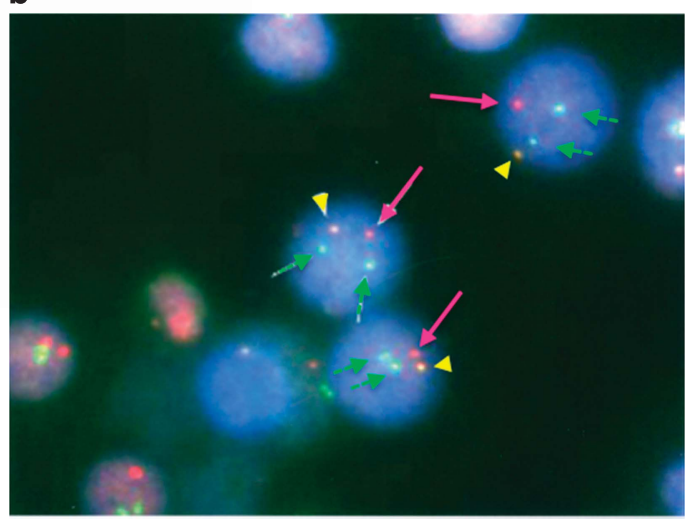

Figure 1. Detection of the EP300-ZNF384 fusion gene. (a) Two split sequences of EP300-ZNF384 obtained from two individual patients by whole transcriptome sequencing were aligned. Complementary DNA showing fusion of the intron following EP300 exon 6 and the intron preceding the first coding exon (exon 3) of ZNF384. Amino-acid sequences of the predicted EP300-ZNF384 fusion protein are presented. Arrows indicate the fusion points. The box indicates the initiation codon of ZNF384. \#, inserted amino acids originating from the $5^{\prime}$ untranslated 5-bp sequence of ZNF384. The predicted EP300-ZNF384 protein is schematically indicated. NRID, nuclear receptor interaction domain; TAZ1, transcriptional adaptor zinc-finger domain 1 (also known as the cysteine-histidine-rich $(\mathrm{CH})$ region $(\mathrm{CH} 1)$ ); $\mathrm{KIX}$, kinase-inducible domain of CREB-interacting domain; Bd, bromodomain; RING, 'really interesting new gene' domain; PHD, plant homeodomain; HAT, histone acetyltransferase domain; ZZ, ZZ-type zinc-finger domain; TAZ2, transcriptional-adaptor zinc-finger domain 2 (the RING-PHD segment is also known as the $\mathrm{CH} 2$ region, and the $\mathrm{ZZ}-\mathrm{TAZ2}$ domain as the $\mathrm{CH} 3$ region); IBiD, IRF3-binding domain; LZ, leucine-rich domain; SR, serine-rich domain; PR, proline-rich domain; NLS, nuclear localization signal; ZFs, Kruppel-type $\mathrm{C} 2 \mathrm{H} 2$ zinc-finger domains; QA, Gln-Ala repeat. (b) The results of FISH using the EP300 probe (green) and ZNF384 probe (red) are indicated. The fusion signals are indicated by yellow arrowheads.

used in this study were obtained from the patients consisted of an uncontinuous series of 68 BCP-ALL without conventional cytogenetic abnormalities and a continuous series of 333 ALL patients, including 291 BCP-ALL patients (133 patients without conventional cytogenetic abnormalities, Supplementary Information). As three out of six cases of EP300-ZNF384+ BCP-ALL (Cases 2-4) belong to later group, the frequency of EP300-ZNF384 expression in BCP-ALL was estimated as $1.03 \%(0.90 \%$ in total ALL, $2.26 \%$ in
BCP-ALL without conventional cytogenetic abnormalities) in our cohort.

EP300 encodes the E1A-binding protein p300 (EP300), a transcriptional co-activator closely related to CREB-binding protein (CBP) and with the ability to interact with a wide spectrum of transcription factors and histone acetyltransferase activity. ${ }^{6}$ On the other hand, the zinc-finger protein 384 (ZNF384) gene encodes a transcription factor that regulates promoters of the extracellular 


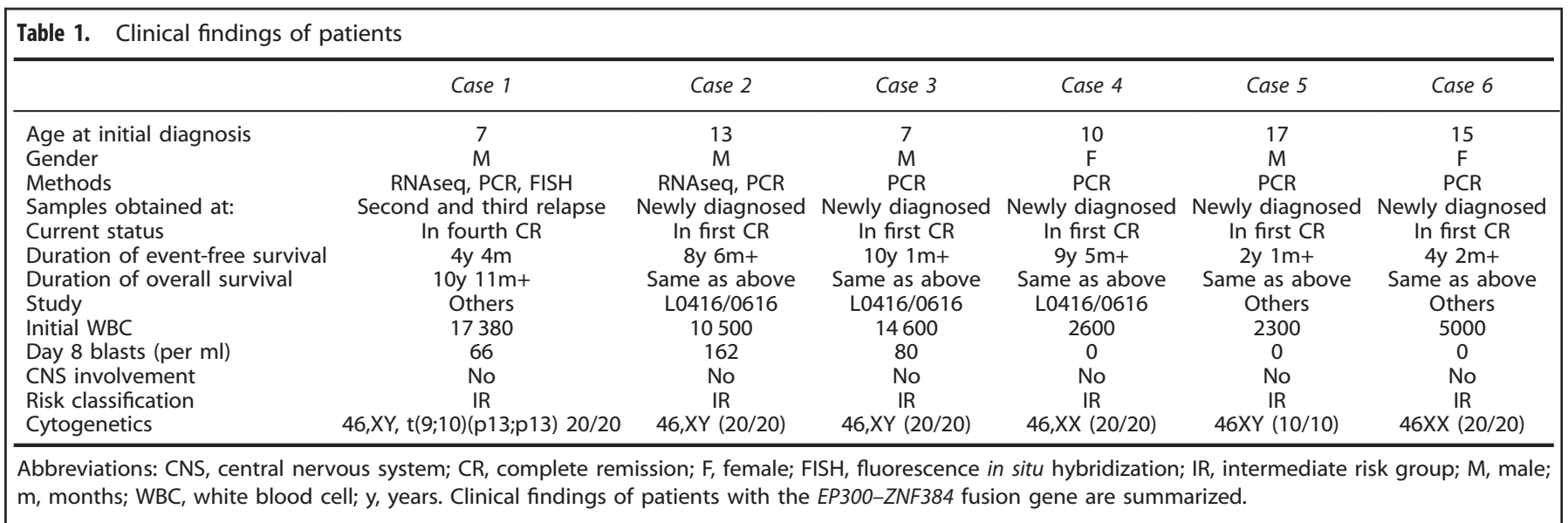

matrix genes. ${ }^{7}$ Chromosomal translocations involving EP300 fused to either Monocytic leukemia zinc-finger protein (MOZ) or Mixed lineage leukemia (MLL) genes are associated with acute myeloid leukemia. ${ }^{8,9}$ ZNF384 is known to be involved in ALL through fusion with the TET family gene, such as the Ewing sarcoma breakpoint region 1 (EWSR1) gene, TATA box binding protein-associated factor (TAF15) and transcription factor 3 (TCF3 or E2A). ${ }^{10,11}$ Although the mutations of both EP300 and ZNF384 are known to be involved in the development of leukemia, this is the first report on the EP300ZNF384 fusion gene.

The resulting sequence of the EP300-ZNF384 fusion gene (Figure 1a) revealed that exon 6 of EP300 was fused to exon 3 (the first coding exon) of ZNF384. The fusion point of ZNF384 is the same as those of previously reported ZNF384-related fusion genes between TAF15 and E2A genes. ${ }^{10,11}$ The 5 -bp sequence upstream of the initiation codon in ZNF384 is included, while the EP300ZNF384 fusion transcripts were predicted to encode a fusion protein with in-frame joining between the $\mathrm{N}$-terminal portion of EP300 and the entire ZNF384 protein. A schematic representation of the EP300-ZNF384 fusion protein predicted to encode a 110$\mathrm{kDa}$ protein with 1027 amino acids ${ }^{10,12}$ is shown in Figure 1a. The EP300-ZNF384 fusion protein retains the transcriptional adapter zinc-finger 1 (TAZ1) domain in the cysteine-histidine-rich region 1 $(\mathrm{CH} 1)$ of EP300, but it lacks the other domains such as the histone acetyltransferase (HAT) domain to be included in EP300. On the other hand, the complete ZNF384 protein is retained in the EP300-ZNF384 fusion.

The clinical findings of EP300-ZNF384 fusion-positive patients are summarized in Table 1. The patients were aged between 7 and 17 years (mean: $11.5 \pm 3.8$ ), comprised four males and two females, and were all classified into an intermediate risk group at the initial diagnosis based on an advanced age. ${ }^{13}$ Their initial white blood cell count ranged from 2300 to 17330 (mean: $8730 \pm 5849$ ). All patients showed a good response to steroid monotherapy, using the cutoff of $1000 / \mu$ l for the blast count in peripheral blood on day $8 .^{13}$ All six patients achieved complete hematological remission after induction therapy. Four patients (Cases 2, 3, 4 and 6) completed the entire protocol and maintained complete remission for 4-10 years. One patient (Case 5) received maintenance therapy and was in complete remission for 2 years and 1 month. The remaining patient (Case 1) also completed the initial therapy, but he relapsed in the off-therapy period, and he is now in his fourth complete remission after a second bone marrow transplantation.

Importantly, the predicted chromosomal translocation $\mathrm{t}(12 ; 22)$ (p13;q13) was not detected in all six cases by conventional G-banding, and five patients (Cases 2-6) showed a normal karyotype on cytogenetic analysis. The remaining patient (Case $1)$, who had relapsed three times, consistently showed $46, \mathrm{XY}, \mathrm{t}$
$(9 ; 10)($ p13;p13) from the initial diagnosis through to the third relapse, whereas corresponding gene fusion was not identified by whole transcriptome sequencing. As FISH confirmed the presence of EP300-ZNF384 translocation in Case 1 and 954 among 1000 cells showed positive fusion signals, it is suggested that translocation leading to EP300-ZNF384 is difficult to detect with conventional G-banding.

All six EP300-ZNF384+ cases revealed dull or negative expression of CD10 based on immunophenotypic examination (Supplementary Figure 2, Supplementary Table). They also exhibited negative expression of cytoplasmic $\mu$ chain. In addition, each case aberrantly expressed one or more myeloid antigen(s), including CD13 and CD33. Therefore, most BCP-ALL patients carrying the EP300-ZNF384 fusion gene may be predictable based on their characteristic immunophenotype.

The EP300-ZNF384 fusion and conventional cytogenetic abnormalities are mutually exclusive. FISH analysis revealed that a vast majority of leukemic blasts express the fusion. The above data suggest the involvement of this fusion gene in the development of BCP-ALL, whereas the functional role of this fusion gene is not yet known. As we present in Figure 1a, the EP300-ZNF384 fusion protein is predicted to lack the HAT domain of EP300. It has been suggested that the deregulation of acetylation could lead to a disruption in the balance between proliferation and differentiation during hematopoiesis and in vivo structure-function analysis of EP300 by others demonstrated that the loss of HAT activity results in an increase in the numbers and proliferative potential of hematopoietic progenitors and stem cells. $^{14}$ Furthermore, it has been reported that some B-cell lymphoma harbor frequent structural alterations inactivating CREBBP or EP300 as a major pathogenetic mechanism, reading to specific defects in acetylation-mediated inactivation of the BCL6 oncoprotein and activation of the p53 tumor suppressor. ${ }^{15}$ Therefore, the N-terminal portion of EP300 without the HAT domains in the EP300-ZNF384 fusion may be involved in the development of ALL by deregulating acetylation, but further functional evaluation is necessary.

In contrast, the complete ZNF384 sequence is retained in the EP300-ZNF384 fusion, being the same as in cases of other ZNF384related recurrent fusions observed in acute leukemia. ${ }^{10,11}$ Both EWSR1-ZNF384 and TAF15-ZNF384 have transforming properties in NIH3T3 cells, but do not alter expressions of known ZNF384 target genes. ${ }^{10}$ The functions of the ZNF384 gene in these fusion genes remain unclear and should also be investigated in the future.

In conclusion, we identified a novel recurrent fusion gene between the EP300 and ZNF384 genes in children with BCP-ALL. Further studies involving a large series of patients should be conducted to elucidate the oncogenic properties of EP300-ZNF384 
and confirm the clinical and biological features of patients with BCP-ALL harboring the EP300-ZNF384 fusion gene.

\section{CONFLICT OF INTEREST}

The authors declare no conflict of interest.

\author{
${ }^{14}$ Department of Pediatrics, The University of Tokyo, \\ Bunkyo-ku, Tokyo, Japan; \\ ${ }^{15}$ Department of Pediatrics, Faculty of Medicine, University of \\ Tsukuba, Tsukuba, Ibaraki, Japan and \\ ${ }^{16}$ Department of Pediatrics, St. Luke's International Hospital, \\ Chuo-ku, Tokyo, Japan \\ E-mail: kiyokawa-n@ncchd.go.jp
}

\section{ACKNOWLEDGEMENTS}

We thank K Itagaki, $\mathrm{H}$ Yagi, K Takeda and K Hayashi for their excellent data management and experimental assistance. We thank all members of the Committees of ALL and of Research and Diagnosis of the TCCSG. We also thank K Hayashi and LSI Medience Corporation for their excellent FISH analysis. This work was supported in part by a Health and Labour Sciences Research Grant (3rd-term comprehensive 10-year strategy for cancer control H22-011), the Grant of the National Center for Child Health and Development (26-20) and the Advanced research for medical products Mining Programme of the National Institute of Biomedical Innovation (NIBIO, 10-41, -42, -43, $-44,-45)$. The above funding sources had no role in the collection, analysis or interpretation of the results, or in the writing of the manuscript and decision to submit it

Y Gocho ${ }^{1,2}$, N Kiyokawa ${ }^{1}, \mathrm{H}_{\text {Ichikawa }}{ }^{3}, \mathrm{~K}$ Nakabayashi $^{4}, \mathrm{~T}$ Osumi ${ }^{5}$

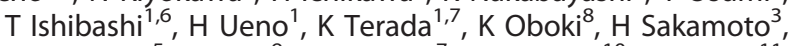
Y Shioda ${ }^{5}$, M Imai $^{9}$, Y Noguchi $^{7}$, Y Arakawa ${ }^{10}$, Y Kojima $^{11}$,

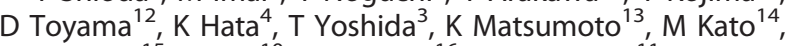
T Fukushima ${ }^{15}, \mathrm{~K} \mathrm{Koh}^{10}$, A Manabe ${ }^{16}$ and A Ohara ${ }^{11}$ from the Tokyo Children's Cancer Study Group

${ }^{1}$ Department of Pediatric Hematology and Oncology Research, National Research Institute for Child Health and Development, Setagaya-ku, Tokyo, Japan;

${ }^{2}$ Department of Pediatrics, Nippon Medical School, Bunkyo-ku, Tokyo, Japan;

${ }^{3}$ Division of Genetics, National Cancer Center Research Institute, Chuo-ku, Tokyo, Japan; ${ }^{4}$ Department of Maternal-Fetal Biology, National Research Institute for Child Health and Development, Setagaya-ku, Tokyo, Japan;

${ }^{5}$ Division of Leukemia and Lymphoma, Children's Cancer Center, National Center for Child Health and Development, Setagaya-ku, Tokyo, Japan

${ }^{6}$ Department of Pediatrics and Adolescent Medicine, Juntendo University School of Medicine, Bunkyo-ku, Tokyo, Japan; ${ }^{7}$ Department of Pediatrics, Japanese Red Cross Narita Hospital, Narita, Chiba, Japan;

${ }^{8}$ Department of Molecular Medical Research, Tokyo Metropolitan Institute of Medical Science Setagaya-ku, Tokyo, Japan; ${ }^{9}$ Department of Pediatrics, Japanese Red Cross Musashino Hospital, Musashino, Tokyo, Japan;

${ }^{10}$ Department of Hematology/Oncology, Saitama Children's Medical Center, Saitama, Japan:

${ }^{11}$ Department of Pediatrics, Toho University Omori Medical Center, Ota-ku, Tokyo, Japan;

${ }^{12}$ Department of Pediatrics, Showa University Fujigaoka Hospital, Yokohama, Kanagawa, Japan;

${ }^{13}$ Department of Allergy and Immunology, National Research Institute for Child Health and Development, Setagaya-ku, Tokyo, Japan;

\section{REFERENCES}

1 Pui CH, Mullighan CG, Evans WE, Relling MV. Pediatric acute lymphoblastic leukemia: where are we going and how do we get there? Blood 2012; 120: 1165-1174.

2 Inaba H, Greaves M, Mullighan CG. Acute lymphoblastic leukaemia. Lancet 2013; 381: 1943-1955.

3 Roberts KG, Morin RD, Zhang J, Hirst M, Zhao Y, Su X et al. Genetic alterations activating kinase and cytokine receptor signaling in high-risk acute lymphoblastic leukemia. Cancer Cell 2012; 22: 153-166.

4 Roberts KG, Li Y, Payne-Turner D, Harvey RC, Yang YL, Pei D et al. Targetable kinase-activating lesions in Ph-like acute lymphoblastic leukemia. N Engl J Med 2014; 371: 1005-1015.

5 Lilljebjörn $\mathrm{H}$, Agerstam $\mathrm{H}$, Orsmark-Pietras $\mathrm{C}$, Rissler $\mathrm{M}$, Ehrencrona $\mathrm{H}_{\text {, }}$ Nilsson $\mathrm{L}$ et al. RNA-seq identifies clinically relevant fusion genes in leukemia including a novel MEF2D/CSF1R fusion responsive to imatinib. Leukemia 2013; 28: 977-979.

6 Blobel GA. CREB-binding protein and p300: molecular integrators of hematopoietic transcription. Blood 2000; 95: 745-755

7 Bidwell JP, Torrungruang K, Alvarez M, Rhodes SJ, Shah R, Jones DR et al. Involvement of the nuclear matrix in the control of skeletal genes: the NMP1 (YY1), NMP2 (Cbfa1), and NMP4 (Nmp4/CIZ) transcription factors. Crit Rev Eukaryot Gene Expr 2001; 11: 279-297.

8 Ida K, Kitabayashi I, Taki T, Taniwaki M, Noro K, Yamamoto M et al. Adenoviral E1A-associated protein p300 is involved in acute myeloid leukemia with $t(11 ; 22)$ (q23;q13). Blood 1997; 90: 4699-4704.

9 Chaffanet M, Gressin L, Preudhomme C, Soenen-Cornu V, Birnbaum D, Pébusque $\mathrm{M} . \mathrm{MOZ}$ is fused to p300 in an acute monocytic leukemia with $t(8 ; 22)$. Genes Chromosomes Cancer 2000; 28: 138-144.

10 Martini A, La Starza R, Janssen H, Bilhou-Nabera C, Corveleyn A, Somers R et al. Recurrent rearrangement of the Ewing's sarcoma gene, EWSR1, or its homologue, TAF15, with the transcription factor CIZ/NMP4 in acute leukemia. Cancer Res 2002; 62: $5408-5412$.

11 Zhong CH, Prima V, Liang X, Frye C, McGavran L, Meltesen L et al. E2A-ZNF384 and NOL1-E2A fusion created by a cryptic $t(12 ; 19)(p 13.3 ; \mathrm{p} 13.3)$ in acute leukemia. Leukemia 2008; 22: 723-729.

12 Delvecchio M, Gaucher J, Aguilar-Gurrieri C, Ortega E, Panne D. Structure of the p300 catalytic core and implications for chromatin targeting and HAT regulation. Nat Struct Mol Biol 2013; 20: 1040-1046.

13 Manabe A, Ohara A, Hasegawa D, Koh K, Saito T, Kiyokawa N et al. Significance of the complete clearance of peripheral blasts after 7 days of prednisolone treatment in children with acute lymphoblastic leukemia: the Tokyo Children's Cancer Study Group Study L99-15. Haematologica 2008; 93: 1155-1160.

14 Kimbrel EA, Lemieux ME, Xia X, Davis TN, Rebel VI, Kung AL. Systematic in vivo structure-function analysis of p300 in hematopoiesis. Blood 2009; 114 4804-4812.

15 Pasqualucci L, Dominguez-Sola D, Chiarenza A, Fabbri G, Grunn A, Trifonov V et al. Inactivating mutations of acetyltransferase genes in B-cell lymphoma. Nature 2011; 471: 189-195.

Supplementary Information accompanies this paper on the Leukemia website (http://www.nature.com/leu) 